\title{
A survey to assess the incidence of Down syndrome risk in rural southwestern Romania
}

\author{
VIRGILIU-BOGDAN SOROP $^{1^{*}}$, ILEANA ENATESCU $^{1 *}$, DANIEL C. MALITA $^{1}$, \\ CAMELIA SZUHANEK ${ }^{2}$, MARIA SOROP FLOREA ${ }^{3}$, LAVINIA BALAN ${ }^{1}$, SINZIANA L. ISTRATE ${ }^{4}$, \\ MADALINA V. BORUGA ${ }^{1}$, DANIELA RADU ${ }^{1}$, DORU M. ANASTASIU ${ }^{1}$ and MONICA SUSAN ${ }^{1}$ \\ ${ }^{1}$ Faculty of Medicine, Department of Radiology and Medical Imaging, and ${ }^{2}$ Faculty of Dental Medicine, \\ Department of Orthodontics, 'Victor Babes' University of Medicine and Pharmacy, 300041 Timisoara; \\ ${ }^{3}$ Faculty of Medicine, Department of Obstetrics and Gynecology, University of Medicine and \\ Pharmacy of Craiova, 200349 Craiova; ${ }^{4}$ Faculty of Medicine, Department of Opthalmology, \\ 'Carol Davila' University of Medicine and Pharmacy, 050474 Bucharest, Romania
}

Received April 13, 2021; Accepted May 13, 2021

DOI: $10.3892 /$ etm.2021.10500

\begin{abstract}
Despite the significant evolution in recent years in the medical field, many fetal conditions that can be detected in the early stages, remain a social and economic burden due to a lack of diagnostic and treatment programs. The main objective of the present study was to realize a screening program related to the early detection of Down syndrome, by analyzing biochemical and imaging markers, in women from the rural areas of Southwest Romania. Accordingly, data from 269 pregnant women were taken into evaluation for maternal age, maternal weight, smoking and diabetic statuses, along with ultrasound measurements that were performed to establish fetal nuchal translucency (FNT) and biochemical analysis of free $\beta$-human chorionic gonadotropin $(\beta$-hCG) and pregnancy-associated plasma protein (PAPP-A). Patients at high risk for trisomy $21(\geq 1: 250)$ were counseled and the optimal protocol was established for each case. Of the 269 patients studied, $5.6 \%$ were included in the risk group based on $\beta$-hCG-associated MoM (multiple median approaches) analysis, sonographic measurements and maternal age correlation. Specifically, $60 \%$ of patients at risk presented a $\beta$-hCG MoM value $>1.5$ and $20 \%$ of patients at risk presented a value $\leq 0.5$ for PAPP-A MoM, and the average maternal age was 33. Measurement of FNT and serum markers, together with associated MoM intervals, was not sufficient to establish the
\end{abstract}

Correspondence to: Dr Daniel C. Malita, Faculty of Medicine, Department of Radiology and Medical Imaging, 'Victor Babes' University of Medicine and Pharmacy, Eftimie Murgu Sq 2, 300041 Timisoara, Romania

E-mail:d.malita@gmail.com

${ }^{*}$ Contributed equally

Key words: sonography, serum, markers, rural area, trisomy 21 diagnosis of trisomy 21 and to make a risk group inclusion. In summary, the association between sonographic measurements and serum marker values, together with maternal age, are predetermined and indispensable conditions for the most accurate classification in a high-risk group.

\section{Introduction}

In the early 1960s, three French scientists (Lejeune, Gautier and Turpin) demonstrated the presence of an additional copy of Hsa21 (human chromosome 21) in the cells of patients with Down syndrome (1). Despite the small number of genes on this chromosome compared to other human chromosomes in the same category, it has not been possible to link the function of specific genes with the defining characteristics of individuals with Down syndrome and intellectual disability, along with other signs and symptoms of this pathology $(2,3)$. There is a network of registries at the European level that aims to assess and epidemiologically monitor congenital anomalies that in the early 2000 s covered approximately $25 \%$ of births in the European Union. Trisomy 21 (Down syndrome) accounted for about 24 cases for every 10,000 births in Europe, for the years 2011-2018 (4). Since the 1980s, the proportion of births to mothers over the age of 35 has increased alarmingly, from 8 to $14 \%$, and by the end of the 1990 s this proportion varied between 10 and $25 \%$, depending on the country and region (5). Cases of pregnancy interruption due to the prenatal diagnosis of Down syndrome, in the member countries of the European Union, for the years 1995-1999, ranged from 0\% (Ireland, Malta; interruption of pregnancy is illegal) to less than $50 \%$ in 14 other regions, with a percentage of $77 \%$ in Paris. With the increase in the average maternal age in Europe there has been an increase in the number of pregnancies affected by Down syndrome and prenatal screening has become a public health issue in these cases, especially due to significant differences in percentages based on geographical regions (5).

The disorder caused by chromosome 21 trisomy is also associated with a number of physical abnormalities and 
intellectual disabilities. More than $40 \%$ of babies diagnosed with Down syndrome have a major heart abnormality, while other major birth defects without heart damage are also common, as is evident in Fig. 1, as presented in EUROCAT registries (6).

Efficient evaluation for trisomy 21 can be made using a combination of maternal age, fetal nuchal translucency (FNT), serum markers such as $\beta$-human chorionic gonadotropin $(\beta-h C G)$ and pregnancy-associated plasma protein-A (PAPP-A) during gestation of $11^{+0}-13^{+6}$ weeks with a detection rate of fetuses with trisomy 21 of over $90 \%$, taking into account the false-positive rate of $5 \%(7,8)$. In the case of risk detection, a solution to increase screening accuracy is to include the evaluation of the nasal bone and blood flow in the ductus venosus and on the tricuspid valve, leading to an improvement of the screening efficiency, towards a detection rate of $93-96 \%$ and a false-positive rate of $2.5 \%$ (9).

The aim of the present study was to screen for a period of four years, pregnant women in south-western Romania from rural areas, through a combination of maternal age, measurement of fetal NT thickness and evaluation of biochemical markers in order to evaluate the incidence of the risk associated with trisomy 21, while emphasizing the need to update databases to highlight cases of trisomy 21 . The percentage variations related to the incidence of Down syndrome from country to country and from area to area are significant. In rural areas from Romania, access to medical services is more limited and the application of screening programs is a solution to contribute to a realistic assessment of the incidence of fetal abnormalities associated with Down syndrome.

\section{Materials and methods}

General. The present study included all determinations obtained from women in a given population who had undergone one or more index tests compared to a reference standard. At the same time, only pregnant women at a gestational age of less than 14 weeks confirmed by ultrasound and who have completed an informed consent form were eligible. Patients who were not in the first trimester of pregnancy and did not reside in rural areas were not eligible for inclusion in the study. Individual ultrasound markers or combinations thereof with one or more tests of serum markers were examined in 269 patients, aged between 16 and 45 years (average age, 30 years) between January, 2015 and December, 2018. None of the patients reported diabetes. Informed consent was obtained from all the patients prior to evaluations and analysis and all data were collected according to the principles of The Declaration of Helsinki. In the case of minor patients, informed consent was obtained from parents or guardians. The study received the approval No. 36/2017 from the Ethics Committee of the 'Victor Babes' University of Medicine and Pharmacy Timisoara, Romania, respecting the standards of personal data protection.

Method. Fetal nuchal translucency (FNT) was examined primarily as an ultrasound marker and serum markers were evaluated as free human chorionic gonadotropin beta $(\beta-h C G)$ and pregnancy-associated plasma protein A (PAPP-A). The current study was based on data from single fetal pregnancies from prospective combined screening in the first trimester for trisomy 21 in the Caransebes, area, Banat region in south-western Romania. Serum samples collected at gestational age between $11^{+0}$ and $13^{+6}$ weeks were analyzed in order to determine free $\beta$-hCG and PAPP-A concentrations using the Time Resolved Amplified Cryptate Emission (TRACE) technology, an immunochemical method based on fluorescence that allows the determination of homogeneous phase analytes, with the help of the KRYPTOR analyser (Brahms, Thermo Scientific). Transabdominal and, when necessary due to visualization problems, transvaginal ultrasound measurements were performed in order to diagnose possible major fetal defects and make specific measurements (e.g., fetal crown length and nuchal translucent thickness). Gestational age was calculated based on CRL (crown-rump length) at the time of FNT measurement. Maternal weight, measured at the time of serum screening, ultrasonographic measurements and biochemical results were recorded in a database. The associated risks largely depended on the accuracy of the data provided by patients, and smoking status and the existence of diabetes, were reported by each patient on a completed personal questionnaire.

The analysis and processing models included the terms from the screening center, gestational age, type of pregnancy (monofetal or not), maternal weight, smoking-related condition and the existence or not of diabetes. Following the results of the analyzes, the patients were divided into two main groups: i) Low risk-a situation in which the value of the calculated risk was below the established cut-offs, but which did not necessarily guarantee the absence of Trisomy 21 (Down syndrome); ii) increased risk-a situation in which the value of the calculated risk exceeded the established cut-offs, did not establish the diagnosis of Trisomy 21 (Down syndrome) but required further investigations.

Statistical analysis. Statistical analyses were performed using IBM SPSS v.27 software and descriptive statistics using Excel from MS Office Pro Plus 2019, respectively. Data are expressed as mean \pm SD. The normality of distributions of biochemical markers was ascertained using the Kolgomorov-Smirnov test; the differences in age, weight, between the other parameters, were assessed by one-way ANOVA followed by Bonferonni's post-hoc comparisons test. Correlations between the studied parameters were examined using Pearson's coefficient. $\mathrm{P}<0.05$ was considered statistically significant.

\section{Results}

Patient characteristics. To obtain an improved diagnostic performance a combination of tests was used including, maternal age and assessment of specific serum markers, or combinations of maternal age, specific serum markers, and sonographic measurements. Individual ultrasound markers or combinations thereof with one or more tests of serum markers were examined in the first trimester, with or without adjustment for maternal age. In the present study, 269 patients were subjected to investigations (by ultrasound and quantification of serum markers-double test), between January 2015 and December 2018 (Table I). The investigation of the correlations between the studied parameters is presented as values of Pearson' coefficient in Table II. 


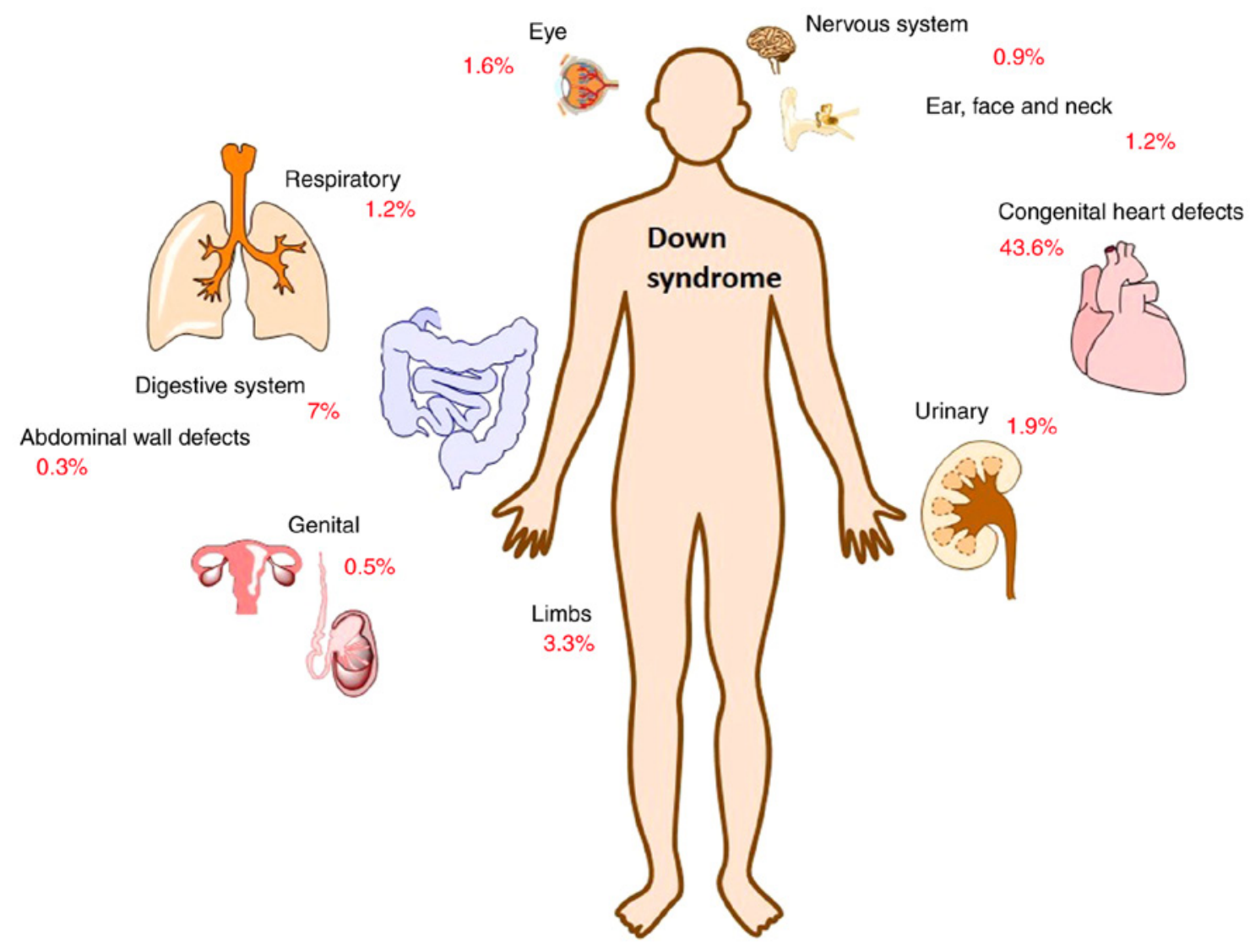

Figure 1. Percentage of cases of Down syndrome, with at least one anomaly, reported by EUROCAT registries.

Distribution of maternal age. Fig. 2 presents the distribution of patients based on their age; the group has been divided in 6 equal sub-groups in order to observe the presence/absence of a normal (gaussian) distribution. Patients under 21 years represent almost $10 \%$, while the age group 21-25 years almost a quarter of the total. The age group 26-30 years are the predominant group (more than 40\%), 31-35 years almost $20 \%, 36-40$ years around $7 \%$ and the last group, $40-45$ years is represented by a percentage of $1.5 \%$ (Fig. 2).

During the four years, correlated with age, eight minors also presented themselves for investigations: Two aged 17 during 2015, three aged 17 during 2016, two aged 16 during 2017 and a 16-year-old during 2018. Regarding the smoking status, out of the 269 patients included in the present study for the years 2015-2018, 31 of them declared themselves smokers while the remaining 238 were non-smokers. None of the patients reported diabetes.

Distribution of FNT. Of the 269 cases studied, a percentage of $5.6 \%$ were included in the risk group $(\geq 1: 250)$ and a percentage of $1.5 \%$ was at the limit (in the risk range 1:251-1:300).

NT was initially implemented to predict the likelihood of a fetus with Down syndrome. Maternal age can be combined with fetal NT and maternal serum biochemistry (free $\beta \mathrm{HCG}$ and PAPP-A) at $11^{+0}$ to $13^{+6}$ weeks to identify approximately $90 \%$ of affected fetuses. Establishing FNT screening programs requires licensed physicians and certified sonographers. In the present study, no values of NT $\geq 3$ were recorded. A single value of 2.9 was recorded during 2015 , the patient was included in a risk group after association with serum markers, age and other factors was taken into consideration. Fig. 3 shows the distribution of fetal nuchal translucency values in the studied group of patients from the rural areas of southwestern Romania.

Distribution of PAPP-A values. PAPP-A values were determined by well-known fluorescence immunochemical methods. There were only 20 MoM values $\leq 0.5$ for PAPP-A which could theoretically signal some associated risks (intrauterine growth restriction, premature birth, preeclampsia, miscarriage or fetal death at $\geq 24$ weeks), as follows: i) During 2015 three values of $0.37,0.48,0.41$ and a value of 0.36 for a 33 -year-old patient who was classified after taking into account all the criteria selected in the risk category; ii) during 2016 two values of 0.4 and 0.36 , but not included in the risk category of trisomy 21 ; iii) during 2017 six values of $0.41,0.49,0.31,0.29,0.47$, and 0.46 , and two included after the analysis of all parameters in the risk group, 0.35 and 0.48 ; and iv) during 2018 six values of $0.31,0.4,0.49,0.4,0.49$ and 0.43 . The graphical representation of the values of pregnancy-associated plasma protein A values in the studied group of patients from the rural areas of southwestern Romania, is shown in Fig. 4.

Distribution of $\beta$-hCG values. $\beta$-hCG values were also determined by well-known fluorescence immunochemical methods. There were $23 \%$ values $\geq 1.5$ and of the 269 patients included in the risk group $60 \%$ had values $\geq 1.5$ of $\beta$-hCG-associated MoM, as follows: i) During 2015 two values of 1.71, 1.64; ii) during 2016 one value of 3.67; iii) during 2017 five values 
Table I. Description of the studied group from the rural areas of southwestern Romania.

\begin{tabular}{lcccccc}
\hline Parameter & Min. value & Max. value & Mean \pm SD & Median & SE & CI 95\% \\
\hline Age, years & 16 & 41 & $27.55 \pm 5.09$ & 27 & 0.31 & $26.94-28.16$ \\
Weight, $\mathrm{kg}$ & 43 & 122 & $65.75 \pm 12.97$ & 63 & 0.79 & $64.20-67.30$ \\
FNT, $\mathrm{mm}$ & 0.70 & 2.90 & $1.57 \pm 0.29$ & 1.60 & 0.02 & $1.54-1.61$ \\
PAPP-A, UI/l & 0.61 & 14.65 & $3.91 \pm 2.65$ & 3.12 & 0.16 & $3.59-4.23$ \\
Free $\beta$-hCG, $\mathrm{ng} / \mathrm{ml}$ & 9.72 & 284.00 & $44.79 \pm 27.30$ & 38.03 & 1.66 & $41.53-48.05$ \\
\hline
\end{tabular}

Table II. Classification of correlations between fetal nuchal translucency, free human chorionic gonadotropin beta and pregnancyassociated plasma protein A (using multiple median approaches), based on the criteria of Colton (10).

\begin{tabular}{lcc}
\hline Correlation type & Direct dependence & Inverse dependence \\
\hline Strong correlations & NT vs. its MoM $(\mathrm{r}=0.900, \mathrm{P}<0.01)$ & \\
& PAPP-A vs. its MoM $(\mathrm{r}=0.778, \mathrm{P}<0.01)$ & - \\
Medium correlations & Free $\beta$-hCG vs. its $\mathrm{MoM}(\mathrm{r}=877, \mathrm{P}<0.01)$ & - \\
Weak correlations & - & PAPP-A vs. weight $(\mathrm{r}=-347, \mathrm{P}<0.01)$
\end{tabular}

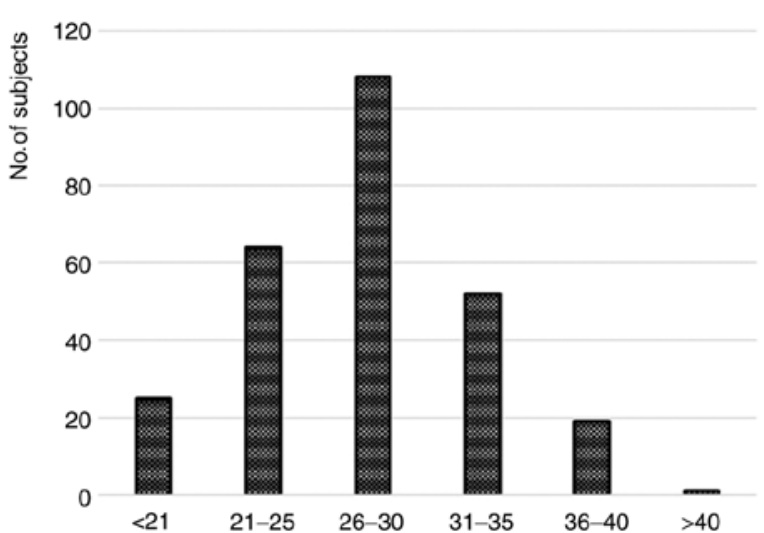

Figure 2. Distribution of maternal age in the studied group of patients from the rural areas of southwestern Romania, between January, 2015 and December, 2018

of $1.89,1.65,1.84,1.95$ and 3.33 ; and iv) during 2018 one value of 4.17. The graphical representation of the values of $\beta$-hCG registered in the period January, 2015-December, 2018, is presented in Fig. 5.

Screening based on maternal age, fetal NT and the two additional serum markers resulted in a detection rate $\geq 85 \%$, and a false-positive rate of $3 \%$.

\section{Discussion}

Screening programs must be implemented through effective health policies and testing of patients in rural areas must be carried out especially taking into account the limited access of this set of individuals to medical services. In the present study, the patients from a rural area from the south-western part of Romania were tested, and of 269 patients, a percentage of $5.6 \%$ were included in the risk group for trisomy 21 . Down

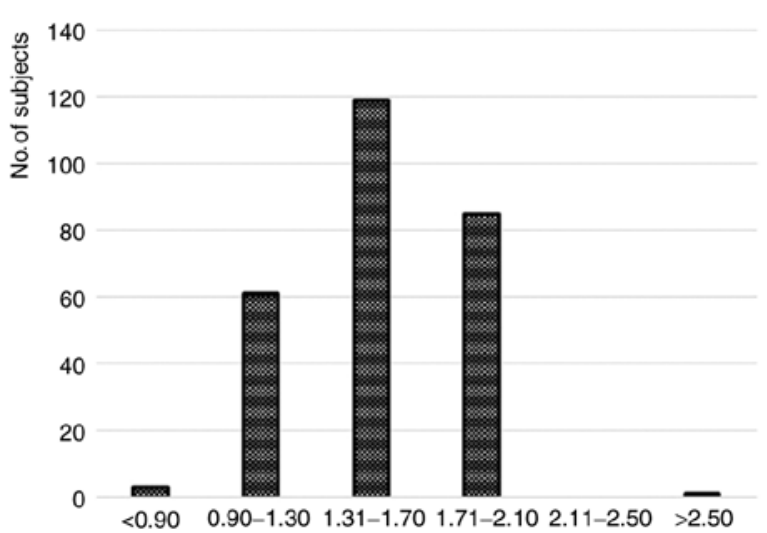

Figure 3. Distribution of fetal nuchal translucency values in the studied group of patients from the rural areas of southwestern Romania, between January, 2015 and December, 2018.

syndrome is an abnormality that does not take into account race and socio-economic status and can occur in any woman, albeit the probability increases in direct proportion to age, especially over 35 years. In modern times, the decision to become a parent has been postponed in favor of a career and increasingly couples are finding themselves at an advanced age when they become parents. Therefore, compared to the 1990s there is an increase in the prevalence of Down syndrome from an average of $16 \%$ to one of $23 \%$ in 2014 . The variation in percentages is significant in different regions of the European Union (11). Initially, the inclusion of pregnancies in the risk category for Down syndrome was realized exclusively according to maternal age (high risk at the age of over 35 years); in 1987, $\beta$-hCG was introduced to detect chromosomal abnormalities; in 1994, PAPP-A was introduced as the second serum marker to detect such abnormalities ( $\beta$-hCG increases significantly and PAPP-A decreases significantly in 


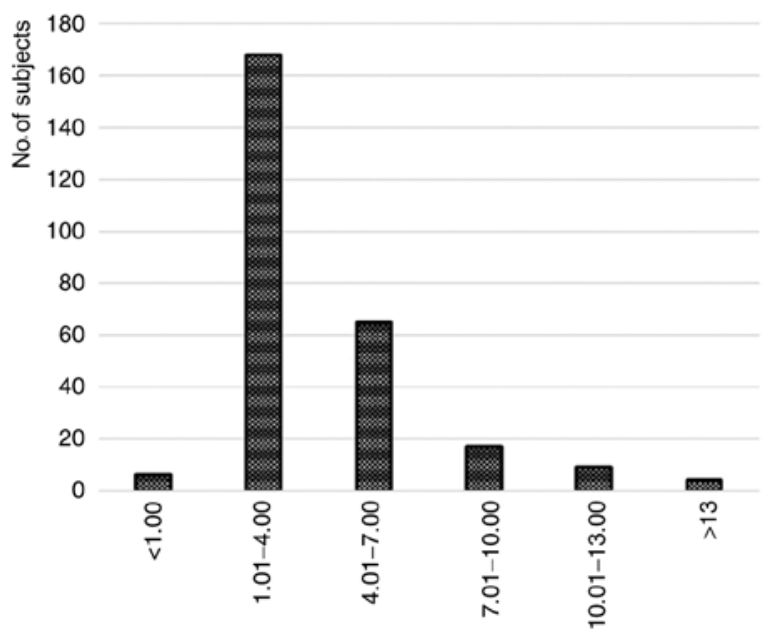

Figure 4. Distribution of pregnancy-associated plasma protein A values in the studied group of patients from the rural areas of southwestern Romania, between January, 2015 and December, 2018.

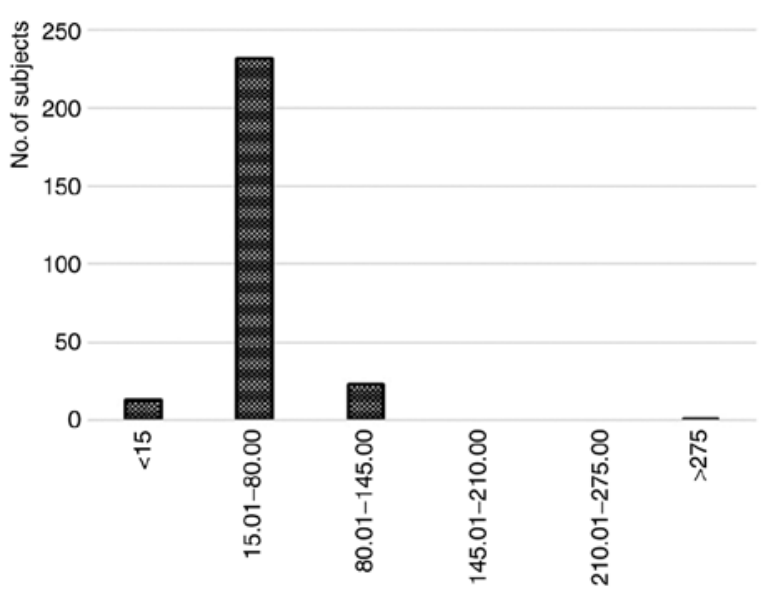

Figure 5. Distribution of free $\beta$-human chorionic gonadotropin values in the studied group of patients from the rural areas of southwestern Romania, between January, 2015 and December, 2018.

pregnancies with trisomy 21) while measurement of NT was introduced as the first sonographic marker in 1992 (12). Since 2007 most revised guidelines have proposed that screening for Down syndrome be conducted regardless of age $(12,13)$.

Sonographic measurements should be made by an experienced person taking into account well-established rules (e.g., median sagittal fetal scan, by appropriate zoom denoting the fetus on three quarters of the screen image showing the profile and upper chest) with differentiation of fetal skin and amniotic membrane and a 'neutral' position of the fetus. Any deviation from these rules translates into erroneous measurements and false-positive results (e.g., in a hyper-extended position NT detection can be overestimated) (14). During the examination, several measurements are performed and the risk calculation is made with the measurement that meets all the mentioned criteria. In most cases (over 95\%) NT measurement is conducted by transabdominal scan; however, in particular instances ( $\leq 5 \%$ of cases) NT measurement must be realized by transvaginal scan (14). NT thickness denotes an increase directly proportional to gestational age, after 14 weeks it is no longer relevant, and the normal range of changes is variable in the literature: A fixed cut off $(2.5,3.0$ or $3.5 \mathrm{~mm})$, a cut-off correlated with fetal CRL $(95-99 \%)$ or by using multiple median approaches (MoM) (15).

A number of clinical and biological factors, including low plasma protein levels associated with plasma pregnancy-A and elevated free human chorionic gonadotropin values, can significantly influence pregnancy outcome. The PAPP-A glycoprotein, one of the four placental antigens identified in the mid-1970s and associated with unknown function, found in high plasma concentrations in pregnant women, which was the name given to pregnancy-associated plasma protein, is produced by the trophoblast sinus that is present in the maternal blood in low concentrations at the beginning of pregnancy and its value increases with gestational age (14). This glycoprotein has an essential role in the development of the placenta, values $<5 \%$ are associated with various obstetric complications, premature labor and low birth weight, and vascular failure, especially in fetuses where intrauterine growth restriction is also correlated with low values (16). PAPP-A is also responsible for IGFBP-4-dependent proteolysis of IGF (insulin-like growth factor) in environments conditioned by normal human fibroblasts, its mRNA is expressed in a significant number of non-pregnancy-related cell and tissue types (17). Currently, protein has attracted attention due to its possible involvement in certain types of cancer, as it improves the action of IGF by specific cleavage of inhibitory insulin-like growth factor binding proteins (IGFBPs), mainly IGFBP-4 which leads to loss of binding affinity for IGF, resulting in significant bioavailability of IGF for IGF-IR-mediated proliferation, survival, and migration, considering the fact that enhanced IGF-IR signaling is associated with tumor growth and metastasis $(18,19)$. The first link between breast cancer and PAPP-A was made in the mid-1980s, and at that time it was considered an independent predictor of early recurrence of stage I breast cancer, but by using a specific monoclonal antibody, the PAPP-A antigen has been shown to be expressed in human breast cancer associated with an aggressive phenotype (20). The $\beta$ subunit of human chorionic gonadotropin $(\beta-\mathrm{hCG})$ is detectable in the plasma of pregnant women from day eight after ovulation, and plasma levels may provide useful information for the following values: In patients with viable intrauterine pregnancy lower $\beta$-hCG levels of $1,500 \mathrm{mIU} / \mathrm{ml}, 1,500-3,000 \mathrm{mIU} / \mathrm{ml}$ or more than $3,000 \mathrm{mIU} / \mathrm{ml}$ will increase after $48 \mathrm{~h}$ by at least 49,40 and $33 \%$, respectively, and a lower growth rate indicates early pregnancy loss or ectopic pregnancy. From the 10th week of gestation, the level of $\beta$-hCG reaches a plateau or decreases (21).

In addition to this, a lot of surveys have been conducted as far as early diagnosis of trisomy disorders is concerned. Tang et al suggested that the reliability of tests is increased when nuchal translucency thickness and ductus venosus blood flow is integrated for the early diagnosis of trisomy 21,18 and 13 for the Western Chinese population (22).

In addition, previous non-invasive prenatal tests (NIPT) have been evolved for the detection of trisomies 13, 18 and 21. Specifically, Zhang and Zhang (23) and Qiang et al (24) showed that the NIPT technique is feasible for the prenatal screening of T18 and T21 with very high sensitivity and specificity and it can be used as an important alternative screening method for Down's syndrome in women $(23,24)$. 
In certain instances, the calculations in the combined tests may reveal a low risk of developing fetal abnormalities, but the numerical values of the serum markers monitored may be suggestively altered compared to the values corresponding to gestational age, in which case these details must be specified. For example, values $\leq 0.5 \mathrm{MoM}$ for PAPP-A may signal a risk associated with intrauterine growth restriction, premature birth, preeclampsia, miscarriage, or fetal death at $\geq 24$ weeks $(25,26)$. At the same time, values $\leq 0.25 \mathrm{MoM}$ in the first trimester of pregnancy can signal a significant risk of pregnancy loss of up to 24 weeks (27).

In the present study, a significant number of patients from the rural area were screened. It is disconcering that only two patients in the risk group were over 40 years of age. Moreover, two patients were under 30 years of age. Data on the incidence of Down syndrome are extremely varied and difficult to access. Patients included in this risk group easily forego further testing due to social status, religion or lack of information. Therefore, the realization of screening programs correlated with counseling and monitoring programs is mandatory. Eastern Europe lacks a database of the incidence of fetal anomalies, probably due to the governing regimes in the Balkan countries. In order to ensure access to investigations for patients in rural areas, it is necessary to implement screening and counseling programs for patients.

In summary, the use of sonographic and serum markers, correlated with maternal age are tools recognized for decades in the early diagnosis of the risk of Down syndrome. In rural areas, access to medical services is limited for a variety of reasons (lack of qualified personnel, social condition, material condition, level of awareness). Thus, screening programs are needed to make an assessment of the incidence of risk of Down syndrome as accurate as possible. In the present study, over $5 \%$ of patients at risk were detected following the screening. Maternal age is a key factor in determining this risk and the values recorded in the study reveal an early age at which these fetal abnormalities can develop. This requires further testing and the development of more extensive screening programs.

\section{Acknowledgements}

Not applicable.

\section{Funding}

No funding was received.

\section{Availability of data and materials}

Authors may make available to the publisher the data and materials presented in the manuscript.

\section{Authors' contributions}

VBS, LB and MSF conceived and designed the study. IE, DCM, MS, DR, CS and SLI were involved in data curation and analysis. VBS, DR, MSF and LB contributed to conducting the experiment, acquired data and confirmed the originality of raw data. MVB, DR, MS, DMA, DCM and SLI were involved in project administration, supervision, visualization and data analysis. DCM and CS were responsible for setting up the software. VBS, CS, MSF and IE wrote the original draft, which was reviewed and edited by DCM, CS and DMA. All authors have read and agreed to the published version of the manuscript.

\section{Ethics approval and consent to participate}

Informed consent was obtained from all the patients prior to evaluations and analysis and all data were collected according to the principles of The Declaration of Helsinki. In the case of minor patients, the informed consent was obtained from parents or guardians. The study was approved by the 'Victor Babes' University of Medicine and Pharmacy Timisoara, Doctoral School (no. 23/5.11.2018) and the Ethics Committee of 'Victor Babes' University of Medicine and Pharmacy Timisoara (no. 36/2017).

\section{Patient consent for publication}

Not applicable.

\section{Competing interests}

The authors declare that they have no competing interests.

\section{References}

1. Menghini D, Costanzo F and Vicari S: Relationship between brain and cognitive processes in Down syndrome. Behav Genet 41: 381-393, 2011

2. Piovesan A, Caracausi M, Antonaros F, Pelleri MC and Vitale L: GeneBase 1.1: A tool to summarize data from NCBI gene datasets and its application to an update of human gene statistics. Database (Oxford) 2016: baw153, 2016.

3. Letourneau A and Antonarakis SE: Genomic determinants in the phenotypic variability of Down syndrome. Prog Brain Res 197: 15-28, 2012.

4. European Commission: EUROCAT Data-Prevalence and Tables. Accessible at: https://eu-rd-platform.jrc.ec.europa. eu/eurocat/eurocat-data/prevalence_en.

5. Dolk H, Loane M, Garne E, De Walle H, Queisser-Luft A, De Vigan C, Addor MC, Gener B, Haeusler M, Jordan H, et al: Trends and geographic inequalities in the prevalence of Down syndrome in Europe, 1980-1999. Rev Epidemiol Sante Publique 53 Spec No 2: 2S87-2S95, 2005.

6. Morris JK, Garne E, Wellesley D, Addor MC, Arriola L, Barisic I, Beres J, Bianchi F, Budd J, Dias CM, et al: Major congenital anomalies in babies born with Down syndrome: A EUROCAT population-based registry study. Am J Med Genet A 164A: 2979-2986, 2014.

7. Kagan KO, Wright D, Baker A, Sahota D and Nicolaides KH: Screening for trisomy 21 by maternal age fetal nuchal translucency thickness, free beta-human chorionic gonadotropin and pregnancy-associated plasma protein-A. Ultrasound Obstet Gynecol 31: 618-624, 2008.

8. Nicolaides KH: Nuchal translucency and other first-trimester sonographic markers of chromosomal abnormalities. Am J Obstet Gynecol 191: 45-67, 2004.

9. Kagan KO, Staboulidou I, Cruz J, Wright D and Nicolaides KH: Two-stage first-trimester screening for trisomy 21 by ultrasound assessment and biochemical testing. Ultrasound Obstet Gynecol 36: 542-547, 2010.

10. Colton T: Statistics in medicine. Little Brown and Company, New York, pp19-20, 1974.

11. Lanzoni M, Kinsner-Ovaskainen A, Morris J and Martin S: EUROCAT-Surveillance of Congenital Anomalies in Europe: Epidemiology of Down syndrome 1990-2014. La Placa G and Spirito L (eds). Publications Office of the European Union, Luxembourg, 2019. 
12. Heidari R, Akbariqomi M, Motevaseli E, Omrani MD, Kooshki H, Shamshiri AR, Shafei S, Absalan M, Mazlomi MA, Saleh Gargari S and Tavoosidana G: Performance and predictive value of first Trimester screening markers for down syndrome in Iranian pregnancies. J Family Reprod Health 12: 121-128, 2018.

13. Royère D; Working Group Trisomy 21 Screening: The impact of introducing combined first-trimester trisomy 21 screening in the French population. Eur J Public Health 26: 492-497, 2016.

14. Cignini P, Maggio Savasta L, Gulino FA, Vitale SG, Mangiafico L, Mesoraca A and Giorlandino C: Predictive value of pregnancy-associated plasma protein-A (PAPP-A) and free beta-hCG on fetal growth restriction: Results of a prospective study. Arch Gynecol Obstet 293: 1227-1233, 2016.

15. Stefanovic V, Äyräs O, Eronen M, Paavonen J and Tikkanen M Clinical utility of nuchal translucency screening. Res Rep Neonatol 4: 169-176, 2014

16. Goetzinger KR, Singla A, Gerkowicz S, Dicke JM, Gray DL and Odibo AO: Predicting the risk of pre-eclampsia between 11 and 13 weeks' gestation by combining maternal characteristics and serum analytes, PAPP-A and free $\beta$-hCG. Prenat Diagn 30 1138-1142, 2010.

17. Conover CA: Key questions and answers about pregnancyassociated plasma protein-A. Trends Endrocrinol Metab 23 242-249, 2012

18. Monget $\mathrm{P}$ and Oxvig C: PAPP-A and the IGF system. Ann Endocrinol (Paris) 77: 90-96, 2016.

19. Pollak M: The insulin and insulin-like growth factor receptor family in neoplasia: An update. Nat Rev Cancer 12: 159-169, 2012

20. Conover CA and Oxvig C: PAPP-A and cancer. J Mol Endocrinol 61: T1-T10, 2018
21. Hendriks E, MacNaughton $\mathrm{H}$ and MacKenzie MC: First trimester bleeding: Evaluation and management. Am Fam Physician 99: 166-174, 2019.

22. Tang Y, Luo H, Mu D, Yang T, Zhu Q, Yang F and Liu G: Early diagnosis of trisomy 21 , trisomy 18 and trisomy 13 using nuchal translucency thickness and ductus venosus blood flow waveform in West China. Mol Med Rep 19: 1349-1355, 2019.

23. Zhang $\mathrm{J}$ and Zhang B: Second-generation non-invasive high-throughput DNA sequencing technology in the screening of Down's syndrome in advanced maternal age women. Biomed Rep 4: 715-718, 2016.

24. Qiang R, Cai N, Wang X, Wang L, Cui K, Wang W, Wang X and Li X: Detection of trisomies 13,18 and 21 using non-invasive prenatal tasting. Exp Ther Med 13: 2304-2310, 2017.

25. Patil M, Panchanadikar TM and Wagh G: Variation of PAPP-A level in the first trimester of pregnancy and its clinical outcome. J Obstet Gynaecol India 64: 116-119, 2014.

26. Lau H, Amarasekara C and Uppal T: Low PAPP-A: What are the clinical implications? Australas J Ultrasound Med 15: 26-28, 2012.

27. Antsaklis P, Fasoulakis Z, Theodora M, Diakosavvas M and Kontomanolis EN: Association of low maternal pregnancy-associated plasma protein a with adverse perinatal outcome. Cureus 11: e4912, 2019.

This work is licensed under a Creative Commons Attribution-NonCommercial-NoDerivatives 4.0 International (CC BY-NC-ND 4.0) License. 